\title{
Advances in chronotherapeutic applications for prevention and treatment of cardiovascular diseases
}

\author{
Hannah Cooke-Ariel ${ }^{1 *}$ and Khalique Ahmed ${ }^{2}$ \\ ${ }^{1}$ Adjunct Professor, Scientific Literacy, Department of Physical Science, Lynn University, USA \\ ${ }^{2}$ Professor of Physical Sciences, Lynn University, Boca Raton, USA
}

A temporal association between circadian variation in certain physiologic factors and prevalence of cardiovascular events has been well described [1,2]. Furthermore, triggers exist which have been shown to increase risk for onset of an acute cardiac event [3]. Recently, an increased interest in cardiovascular chronotherapeutics has emerged as the need for more dynamic treatment and prevention strategies has been realized. Researchers are studying pharmacological technology and dosing strategies to prevent cardiovascular events.A review of these findings areherein presented. In addition, recommendations for applications of these technologies are offered.

\section{Chronobiology}

The discipline of study focusing on biological rhythms and their fluctuations is referred to as Chronobiology. There are at least three types of biological rhythms in our bodies. The most commonly occurring are ultradian, infradian and circadian rhythms. Ultradian rhythms have a periodicicity of less than 24 hours. Infradian rhythms have a periodicity greater than 24 hours. Circadian rhythms represent the most frequently occurring cycle, approximating the 24 hour day. The term circadian originated with Franz Halberg, derived from the Latin word "circa" meaning around, and "diem" meaning about a day. Biological rhythms are regulated primarily by the release of melatonin from the pineal gland of the suprachiasmatic nucleus. The level of melatonin activates cortisol release via a negative feedback mechanism, which is also affected by sunlight exposure and sleep-wake cycles. Cortisol released in response to awakening creates a cascade of physiologic changes including increased circulating norepinephrine levels, leading to increased platelet reactivity, increased tissue plasminogen activator inhibition, increased blood viscosity, increased vasomotor tone, elevations in blood pressure and elevations in blood glucose. A delicate balance exists between activation of these physiologic factors at time of awakening, and increased vulnerability for cardiac events.

Blood pressure exhibits a diurnal rhythm superimposed on a circadian pattern, with a bi- modal distribution cresting in early morning hours and again in the evening. Much attention has been directed toward understanding the dynamic physiologic changes and underlying pathophysiology of adverse cardiovascular events. Preventive strategies recently advanced in chronotherapy have validated the benefits of dynamic dosing strategies and interventions.

\section{Chronotherapy of cardiovascular disease}

Based on the vast knowledge of circadian patterns in frequency and risk of cardiovascular events, intuitively, dosing strategies targeting these high risk periods would seem ideal. However, despite the mounting knowledge of the causal relationship between circadian variation and frequency of cardiac events, it has only been recently that studies evaluating Chronotherapeutic dosing strategies have become available [4-8].

Investigations into the pharmacodynamics of the angiotensin converting enzyme inhibitors and angiotensin receptor blockers reveal greater efficacy when these agents are administered before bedtime. The rationale for this dosing strategy is rooted in the physiologic finding that renin and angiotensin release is more pronounce during sleeping hours, with a surge in activity appearing upon awakening. Studies evaluating the bedtime administration of these classes of agents demonstrate lower bedtime blood pressure means and lower morning blood pressures, a time deemed as a high risk period. In addition, dosing at bedtime led to normalization of the blood pressure curve for twenty-four hours, restoring the dipping pattern observed in normotensive individuals. With this Chronotherapeutic intervention, cardiovascular and cerebral risk is reduced. Similar beneficial effects were observed with at bedtime administration of calcium channel blockers, with significantly less peripheral and pedal edema occurring in patients receiving dihydropyridine derivative calcium channel blockers.

Considering the circadian variation in platelet production, with peaks during sleep, administration of the anti platelet agent aspirin should be expected to exhibit greater overall antiplatelet activity when administered at bedtime. The administration of $80 \mathrm{mg}$ of aspirin at bedtime may further reduce overall cardiovascular burden currently observed with aspirin administration. Also, distribution of aspirin throughout the body would occur toward the early morning hours, providing greater protection versus early morning increases in platelet adhesion and blood viscosity.

For individuals receiving multiple medications, beta-adrenergic blockers may be best administered during the day when heightened adrenergic tone predominates.

Chronotherapeutics offers a paradigmatic approach to improving patient outcomes through dynamic dosing strategies which are based on genetically controlled biological rhythms. This represents a new frontier in medicine which may improve outcomes in individuals receiving cardiovascular pharmacology. Practitioners should continue to hone

Correspondence to: Hannah Cooke-Ariel, Pharm.D, Adjunct Professor, Scientific Literacy, Department of Physical Science, Lynn University, Boca Raton, Florida 33431, USA, Tel: 561-313-8456; E-mail: HAriel@lynn.edu

Received: March 20, 2015; Accepted: April 10, 2015; Published: April 14, 2015 
skills and perspectives on managing cardiovascular hemodynamics, with chronotherapeutics in mind.

\section{Acknowledgment}

Thank you to the Lynn University Dialogue of Scientific Literacy Student Participants.

\section{References}

1. Muller JE, Ludmer PL, Willich SN, Tofler GH, Aylmer G, et al. (1987) Circadian variation in frequency of sudden cardiac death. Circulation 75: 131-8. [Crossref]

2. Muller JE, Stone PH, Turi ZG, Rutherford JD, The MILIS study groups, et al. (1985) Circadian variation in the frequency of onset of acute myocardial infarction. $N$ Engl $J$ Med 313: 1315-1322. [Crossref]
3. Mittleman MA, Mostofsky E (2011) Physical, psychological and chemical triggers of acute cardiovascular events: preventive strategies. Circulation 124: 346-359. [Crossref]

4. Hennekens CH, Dalen JE (2013) Aspirin in the treatment and prevention of cardiovascular disease: past and current perspectives and future directions. Am J Med 126: 373-378. [Crossref]

5. Hennekens $\mathrm{CH}$ (2001) Current perspectives on lipid lowering with statins to decrease risk of cardiovascular disease. Clin Cardiol 24: II-2-5. [Crossref]

6. Hebert PR, Pfeffer MA, Hennekens CH (2002) Use of statins and aspirin to reduce risks of cardiovascular disease. J Cardiovasc Pharmacol Ther: 777- 780.

7. Hennekens CH, Schneider WR, Pokov A, Hetzel S, Demets D, et al. (2010) A randomized trial of aspirin at clinically relevant doses and nitric oxide formation in humans. J Cardiovasc Pharmacol Ther 15: 344-348 [Crossref].

8. Hermida RC, Ayala DE, Fernández JR, Mojón A, Smolensky MH, et al. (2013) Administration-time differences in effects of hypertension medications on ambulatory blood pressure regulation. Chronobiol Int 30: 280-314. [Crossref]

Copyright: $(02015$ Cooke-Ariel H. This is an open-access article distributed under the terms of the Creative Commons Attribution License, which permits unrestricted use, distribution, and reproduction in any medium, provided the original author and source are credited. 